\title{
Co-occurring Hydrocephalus and Polygenic Risk in Autism Spectrum Disorder: A Danish Population- based Cohort Study
}

Tina Nørgaard Munch ( $\nabla$ tina.noergaard.munch@regionh.dk)

Copenhagen University Hospital https://orcid.org/0000-0001-5938-000X

Paula Hedley

Statens Serum Institut

Christian Munch Hagen

Statens Serum Institut

Marie Bækvad-Hansen

Statens Serum Institut

Jonas Bybjerg-Grauholm

Statens Serum Institut

Jakob Grove

Aarhus Universitet

Merete Nordentoft

Region Hovedstadens Psykiatri

Anders D. Børglum

Aarhus Universitet

Preben B. Mortensen

Aarhus Universitet

Thomas M. Werge

Region Hovedstadens Psykiatri

Mads Melbye

Stanford University School of Medicine

David M. Hougaard

Statens Serum Institut

Michael Christiansen

Statens Serum Institut

\section{Research}

Keywords: Autism Spectrum Disorder, Hydrocephalus, Epidemiology, Polygenic Risk Score, Early 
Posted Date: July 17th, 2020

DOl: https://doi.org/10.21203/rs.3.rs-41560/v1

License: (c) (i) This work is licensed under a Creative Commons Attribution 4.0 International License. Read Full License

Loading [MathJax]/jax/output/CommonHTML/jax.js 


\section{Abstract \\ Background}

The association between autism spectrum disorder and hydrocephalus is not well understood, despite demonstrated links between autism spectrum disorder and cerebrospinal fluid abnormalities. Based on the hypothesis that autism spectrum disorder and hydrocephalus may, at least in some cases, be two manifestations of a shared congenital brain pathology, we investigated the potential association between autism spectrum disorder and hydrocephalus in a large Danish population-based cohort, and whether the polygenic risk scores for autism spectrum disorder changed as a function of the presence of hydrocephalus.

\section{Methods}

Patients and controls were obtained from the Lundbeck Foundation Initiative for Integrative Psychiatric Research iPSYCH2012 case-cohort, which includes all patients with selected psychiatric disorders born in Denmark 1981-2005 along with randomly selected population controls (end of follow-up, December 31, 2016). The associations between individual psychiatric disorders and hydrocephalus were estimated using binary logistic regression with adjustment for age and sex. Polygenic risk scores for autism spectrum disorder were used to compare the genetic architecture of autism spectrum disorder as a function of the presence of hydrocephalus.

\section{Results}

The cohort consisted of 86,571 individuals, of which 14,654 were diagnosed with autism spectrum disorder, 28,606 were population controls, and the remaining were diagnosed with other psychiatric disorders. We identified 201 hydrocephalus cases; 68 among autism spectrum disorder patients and 40 among controls (OR 3.77, 95\% Cl 2.48-5.78). The autism spectrum disorder-hydrocephalus association was significant over the entire subgroup spectrum of autism spectrum disorder. The presence of hydrocephalus did not markedly influence the polygenic risk scores in patients with autism spectrum disorder, which may indicate overlapping genetic architectures or other common aetiology.

\section{Conclusions}

Given the very strong association, we suggest that patients with autism spectrum disorder should be evaluated for co-occurring hydrocephalus on a routine basis as timely neurosurgical intervention is important. Further clarification of the genetic aetiology of both diseases, may help in elucidating shared genetic pathways between autism spectrum disorder and hydrocephalus, and it may elucidate the role of abnormal CSF dvnamics in the pathogenesis of autism spectrum disorders.

Loading [MathJax]/jax/output/CommonHTML/jax.js 


\section{Background}

In Denmark, the setting for this study, the prevalence of autism spectrum disorder in children is reported to be $68.5 / 10,000(1)$ with a male: female ratio of $4: 1$.(2) Characterized by impairments in social interaction, communication, and repetitive or restrictive behaviors,(3) autism spectrum disorder is associated with a plethora of comorbidities, often neurodevelopmental in nature. $(4,5)$ Shen et al. showed in different MRI studies that children with autism spectrum disorder, up to preschool age, consistently exhibit increased volumes of CSF in the subarachnoid space, as compared to other children, a condition also known as external hydrocephalus.(6-9)

Hydrocephalus is an aetiologically heterogeneous neurological condition with an excessive amount of intracranial CSF relative to the brain volume, which occurs in 1.08/1,000 children up to two years of age in Denmark.(10) A relatively common presentation is the Benign Enlargement of the Subarachnoid Space (BESS) or benign external hydrocephalus, that is asymptomatic and usually resolves spontaneously before the child reaches the age of two years. $(11,12)$ Most hydrocephalus patients though, present with symptoms, but not necessarily the well-known acute symptoms of increased intracranial pressure or an obviously increased head circumference. They may just as well be presenting with normal or only slightly increased head circumference, and variable degrees of psycho-motoric delay or failure to thrive during infancy. When neurosurgical treatment is required, they undergo endoscopic fenestration of the third ventricle or insertion of a ventriculoperitoneal shunt.

Although children with hydrocephalus needing surgical treatment, remarkedly often display symptoms compatible with autism spectrum disorder, only one population-based study has investigated this potential association. Thus, Lindquist et al. reported that autism was present in nine out of the 107 children with hydrocephalus (13\%) born in western Sweden during the period 1989-1993.(13)

Genetics play a key role in the aetiology of autism spectrum disorder,(14) which is considered a polygenic disease. Autism spectrum disorder exhibits a high level of heritability,(15) and is considered a polygenic disorder, involving $100+$ genes and, at the time of writing, five GWAS defined loci have been identified. (14) Likewise, childhood hydrocephalus displays a complicated genetic background involving $100+$ genes, $(16,17)$ however much less investigated than the genetic background of autism spectrum disorder.

Based on the hypothesis that autism spectrum disorder and hydrocephalus may, at least in some cases, be two manifestations of a shared congenital brain pathology, we investigate 1) the potential association between autism spectrum disorder and hydrocephalus in a large Danish population-based cohort, 2) whether the polygenic risk scores for autism spectrum disorder are different in autism spectrum disorder patients with and without hydrocephalus, and in hydrocephalus patients without autism spectrum disorder, respectively, and 3) the associations between hydrocephalus and other psychiatric disorders, including attention deficit and hyperactivity disorder.

\section{Methods}

Loading [MathJax]/jax/output/CommonHTML/jax.js 


\section{Data sources}

This register-based case-cohort study is a sub-study of the Lundbeck Foundation Initiative For Integrative Psychiatric Research (iPSYCH, http:www.iPSYCH.au.dk) and combines data from The National Patient Register,(18) The Danish Neonatal Screening Biobank,(19) and the Danish Psychiatric Central Research Register.(20) The registers contains information about all in-patient admissions since 1969, and outpatient visits from 1995 onwards. The data were linked on an individual level using the unique personal identification number from the Danish Civil Registration System.(21) autism spectrum disorder-polygenic risk scores were generated using the Psychiatric Genomics Consortium-autism spectrum disorder dataset, which, similar to this study, is based on the iPSYCH cohort.(22)

\section{Cohort}

Patients and controls were obtained from the Lundbeck Foundation Initiative for Integrative Psychiatric Research iPSYCH2012 case-cohort,(23) which comprises information on individuals with psychiatric disorders $(n=57,875)$ as well as a randomly sampled control cohort from the same source population as the psychiatric cases $(n=28,606)$. Thus, the controls represent the background population. Cases and the population cohort were selected from a study base of 1,472,762 singleton births between May 1, 1981, and December 31, 2005, who were alive and resided in Denmark at their first birthday. End of follow-up was December 31, 2016. Hence, the cohort members were followed for at least 11 years and up to 34 years and 8 months. Cases were defined as all individuals registered with a diagnosis of five selected psychiatric disorders; schizophrenia, bipolar disorder, major depressive disorder, autism spectrum disorder, and attention deficit and hyperactivity disorder.

\section{Variables}

The outcome variables were a registered diagnosis of at least one of the psychiatric disorders and/or a registered diagnosis of hydrocephalus.

\section{Ascertainment of psychiatric disease}

The study base was linked, through the personal identification number, to the Danish Psychiatric Central Research Register to obtain information about psychiatric disorders, defined by the ICD-10 codes: F20 (schizophrenia (SCZ)), F30-F31 (bipolar disorder (BD)), F32-F39 (major depressive disorder (MDD)), F84 (autism spectrum disorder (ASD)), or F90 (attention deficit hyperactivity disorder (ADHD)).(24)

ASD was furthermore divided into the subgroups defined in the ICD-10 system: F84.0 (childhood autism), F84.1 (atypical autism), F84.5 (Asperger's syndrome), F84.8 (other pervasive developmental disorder), and F84.9 (pervasive developmental disorder, unspecified). The latest update of diagnostic information and vital status was December 31, 2016.

In cases where more than one condition was diagnosed, the diagnoses were prioritized with respect to their rank in the ICD-10 code system. Thus, for this study, each individual was only captured within one 
disorder > autism spectrum disorder > attention deficit and hyperactivity disorder. Therefore, the case numbers of the psychiatric disorders are somewhat different from the case numbers of the iPSYCH cohort described by Pedersen et al., in which the diagnoses were not ranked.(23)

\section{Ascertainment of hydrocephalus}

The study base was linked, through the personal identification number, to The National Patient Register. Hydrocephalus cases were defined as having one of the following ICD codes. ICD-10: Q030 (malformation of the cerebral aqueduct of Sylvius), Q030A (atresia of the cerebral aqueduct of Sylvius), ) Q030B (congenital stenosis of the cerebral aqueduct of Sylvius), Q031 (atresia of the median and lateral aperture of the fourth ventricle (foramen of Magendie and Luschka)), Q031A (atresia of the lateral aperture of the fourth ventricle (foramen of Luschka)), Q031C Dandy-Walker malformation, Q038 (congenital hydrocephalus, other forms), Q038A (congenital hydrocephalus, communicating), Q038B (congenital hydrocephalus, external), Q038C (congenital hydrocephalus, internal), G910 (communicating hydrocephalus), G911(obstructive hydrocephalus), G91.3 (hydrocephalus, posttraumatic), G918 (other types of hydrocephalus), G919 (unspecific hydrocephalus).

ICD-8: 34793 (hydrocephalus, acquired internal), 34794 (hydrocephalus, acquired external), 34795 (hydrocephalus, acquired non-specific), 74200 (congenital hydrocephalus, external), 74201 (congenital hydrocephalus, internal), 74209 (congenital hydrocephalus), and 74208 (congenital hydrocephalus, other forms).

\section{Statistical analysis}

The risks of hydrocephalus among patients with psychiatric disorders compared to a random healthy control sample were estimated as odds ratios (OR's) using logistic regression analysis including age and sex as covariates. A p-value of 0.05 was considered significant and Bonferroni correction for multiple testing was performed where appropriate. For groupings with 30 + hydrocephalus positive cases, a sampling distribution of the test statistic of the logit model of the hydrocephalus status was resampled $25 \mathrm{~K}$ times and the coefficients for hydrocephalus extracted from the logit model and compared to the observed. The $p$-values were computed as two-sided. As the number of hydrocephalus positive observations within the groupings were relatively low, the statistical tests were confirmed by a Fisher's exact test (data not shown). Wilcoxon rank sum test was used to compare median age at diagnosis of hydrocephalus among controls and each of the psychiatric disorders.

Autism spectrum disorder-polygenic risk scores were generated using the Psychiatric Genomics Consortium-autism spectrum disorder dataset,(22) following removal of genome distribution outliers based on principal components analysis as detailed in Grove et al., 2019,(14) and a p-value threshold of 0.1. The autism spectrum disorder-polygenic risk score load across the subgroups; hydrocephalus without autism spectrum disorder, autism spectrum disorder without hydrocephalus and autism spectrum disorder in combination with hydrocephalus was expressed as the coefficients from a linear regression of the normalized spectrum disorder-polygenic risk score on the subtypes. The Wald test was used to test 


\section{Results}

Basic characteristics of the cohort are presented in Table 1, including the numbers of patients with each of the five psychiatric disorders, median age at diagnosis, and by the end of follow-up. Among the 86,481 cohort members we identified 201 hydrocephalus cases, distributed as follows: 40 among the 28,606 population controls; 68 among the 14,654 patients with autism spectrum disorder (OR $3.77,95 \% \mathrm{Cl} 2.48-$ 5.78, $p \leq 4 \times 10^{-5}$, and 37 among the 13,901 patients with attention deficit and hyperactivity disorder (OR $1.91,95 \% \mathrm{Cl} 1.20-3.04, \mathrm{p}=0.07$ ), as presented in Table 2 . The remaining 56 cohort members with hydrocephalus were diagnosed with either major depressive disorder, bipolar disorder, or schizophrenia, but no significant associations were found between hydrocephalus and these disorders.

Table 1

Basic characteristics of the study population.

\begin{tabular}{|c|c|c|c|c|}
\hline & $\mathbf{N}$ & $\begin{array}{l}\text { Median age in } \\
\text { years, Dec 2016 } \\
\text { (Interquartile range) }\end{array}$ & $\begin{array}{l}\text { Male } \\
(\%)\end{array}$ & $\begin{array}{l}\text { Median age at diagnosis in years } \\
\text { (Interquartile range) }\end{array}$ \\
\hline $\begin{array}{l}\text { No psychiatric } \\
\text { disease (controls) }\end{array}$ & 28,606 & $22.6(16.9-28.6)$ & 50.4 & Not relevant \\
\hline Schizophrenia & 5,242 & $28.6(25.3-32.2)$ & 53.3 & $21.2(18.9-24.0)$ \\
\hline $\begin{array}{l}\text { Major depressive } \\
\text { disorder }\end{array}$ & 22,868 & $28.8(25.0-32.2)$ & 31.9 & $20.0(17.0-23.3)$ \\
\hline Bipolar disorder & 2,300 & $29.7(26.5-32.8)$ & 35.6 & $22.9(20.0-26.5)$ \\
\hline $\begin{array}{l}\text { Autism spectrum } \\
\text { disorder }\end{array}$ & 14,564 & $19.8(16.2-23.9)$ & 80.9 & $9.6(6.4-13.3)$ \\
\hline Childhood autism & 4,447 & $17.9(14.6-21.6)$ & 81.4 & $7.3(4.8-12)$ \\
\hline Atypical autism & 1,803 & $19.7(16.2-23.6)$ & 75.8 & $10.8(7.1-14.4)$ \\
\hline Asperger's syndrome & 4,405 & $21.7(17.7-25.9)$ & 83.6 & $11.5(8.6-14.9)$ \\
\hline Other PDD & 1,868 & $20.7(16.9-24.5)$ & 80.9 & $10.5(7.6-13.5)$ \\
\hline Unspecified PDD & 2,131 & $19.7(15.9-24.5)$ & 78.5 & $9.5(6.6-13.2)$ \\
\hline $\begin{array}{l}\text { Attention deficit } \\
\text { hyperactivity } \\
\text { disorder }\end{array}$ & 12,901 & $21.2(16.8-25.7)$ & 75.2 & $11.4(8.4-17.0)$ \\
\hline \multicolumn{5}{|c|}{ a Pervasive developmental disorder. } \\
\hline
\end{tabular}


Table 2

Associations between hydrocephalus and five psychiatric disorders.

\begin{tabular}{|llllll|}
\hline & \multicolumn{2}{l}{$\begin{array}{l}\text { Hydrocephalus } \\
\text { (N= 201) }\end{array}$} & \multicolumn{2}{l|}{$\begin{array}{l}\text { Logistic regression adjusted for age } \\
\text { and sex }\end{array}$} \\
\hline Condition / ICD10 code. & $\begin{array}{l}\text { Present } \\
\text { N }\end{array}$ & Absent N & OR & $95 \%$ Cl & p-value $^{\mathrm{a}}$ \\
\hline No psychiatric disease (controls) & 40 & 28,566 & 1 & Reference & Reference \\
\hline Schizophrenia & 13 & 5,229 & 2.01 & $(1.00-3.83)$ & 0.51 \\
\hline Major depressive disorder & 40 & 22,828 & 1.22 & $(0.75-1.99)$ & 1.0 \\
\hline Bipolar disorder & 3 & 2,297 & 1.06 & $(0.25-3.08)$ & 1.0 \\
\hline Autism spectrum disorder & 68 & 14,586 & 3.77 & $(2.48-5.78)$ & $<4 \times 10^{-5}$ \\
\hline $\begin{array}{l}\text { Attention deficit and hyperactivity } \\
\text { disorder }\end{array}$ & 37 & 12,864 & 1.91 & $(1.20-3.04)$ & 0.07 \\
\hline a Bonferroni corrected for multiple tests (here five). & & & & \\
\hline
\end{tabular}

The strong association between hydrocephalus and autism spectrum disorder and the borderline association with attention deficit and hyperactivity disorder were both supported by permutation testing (Supplementary Fig. 1). Furthermore, we investigated the associations between each of the clinical subgroups of autism spectrum disorder and hydrocephalus, as presented in Table 3.

Table 3

Associations between hydrocephalus and clinical subgroups of autism spectrum disorders.

\begin{tabular}{|c|c|c|c|c|c|}
\hline \multirow[b]{2}{*}{ Condition } & \multicolumn{2}{|c|}{ Hydrocephalus } & \multicolumn{3}{|c|}{$\begin{array}{l}\text { Logistic regression adjusted for age } \\
\text { and sex }\end{array}$} \\
\hline & $\mathrm{Nresent}_{\mathrm{N}}$ & $\stackrel{\text { Absent }}{N}$ & OR & $(95 \% \mathrm{Cl})$ & p-value ${ }^{a}$ \\
\hline No psychiatric disease (controls) & 40 & 28,566 & 1 & Reference & Reference \\
\hline Childhood autism & 23 & 4,424 & 3.71 & $(2.08-6.51)$ & $3 \times 10^{-5}$ \\
\hline Atypical autism & 13 & 1,790 & 5.58 & $(2.81-0.40)$ & $1.0 \times 10^{-6}$ \\
\hline Asperger's syndrome & 15 & 4,390 & 2.88 & $(1.49-5.31)$ & 0.005 \\
\hline $\begin{array}{l}\text { Other pervasive developmental } \\
\text { disorder }\end{array}$ & 8 & 1,860 & 3.35 & $(1 \cdot 41-7 \cdot 02)$ & 0.014 \\
\hline $\begin{array}{l}\text { Pervasive developmental disorder, } \\
\text { unspecified }\end{array}$ & 9 & 2,122 & 3.46 & $(1.53-7.01)$ & 0.006 \\
\hline
\end{tabular}


In all five subgroups, we found a significant association with hydrocephalus: Childhood autism (OR 3.71, $95 \% \mathrm{Cl} 2.08-6.51$ ), Atypical autism (OR 5.58, 95\% Cl 2.81-10.4), Asperger's syndrome (OR $2.88,95 \% \mathrm{Cl}$ 1.49-5.31), Other pervasive developmental disorders (OR 3.35, 95\% Cl 1.41-7.02), and Pervasive developmental disorders, unspecified (OR $3.46,95 \% \mathrm{Cl} 1.53-7.01)$.

\section{Polygenic Risk Scores}

In order to investigate if the strong association between hydrocephalus and autism spectrum disorder, both exhibiting complex genetic aetiologies, could rely on a shared genetic aetiology, the autism spectrum disorder-polygenic risk scores were estimated for the hydrocephalus patients with available genetic data. This involved no selection criteria among the hydrocephalus patients and was completely random. We would expect the autism spectrum disorder-polygenic risk score load to change significantly, according to the presence of hydrocephalus, if the genetic aetiology of the two conditions were different. Autism spectrum disorder-polygenic risk scores could be estimated for 154 hydrocephalus patients, as presented in Fig. 1. The standardized autism spectrum disorder-polygenic risk score load was profiled over the three possible combinations of autism spectrum disorder and hydrocephalus, i.e. hydrocephalus with autism spectrum disorder $(n=52)$, hydrocephalus without autism spectrum disorder $(n=102)$, and autism spectrum disorder without hydrocephalus $(n=11,716)$. The reference group consisted of control individuals without autism spectrum disorder or hydrocephalus $(n=21,848)$. No significant group effect was found ( $p>0.85$, Wald test of equal group effect). However, due to the relatively low number of hydrocephalus cases, the statistical power is limited and only a major difference in autism spectrum disorder-polygenic risk in the presence of hydrocephalus would be detectable.

\section{Sub analyses}

A description of age at diagnosis and gender distribution of the hydrocephalus patients by co-occurring psychiatric disorders compared to hydrocephalus patients without psychiatric disorders are presented in Supplementary table 1. Only patients with schizophrenia and major depressive disorder were diagnosed with hydrocephalus significantly later than the controls without co-occurring psychiatric disorders. Thus, hydrocephalus patients with autism spectrum disorder was diagnosed at a median age of 1.0 year (IQR $0.3-5.3$ years), which was not significantly different from the median age of hydrocephalus diagnosis among controls without psychiatric disorders (2.3 years, IQR $0.3-12.1, \mathrm{P}=0.4)$.

\section{Discussion}

This is the first demonstration of a strong co-occurrence of autism spectrum disorder and hydrocephalus in a large population-based cohort (OR: $3.77,95 \% \mathrm{Cl} 2.48-5.78)$. This association was significant for the entire subgroup spectrum of autism spectrum disorder. In absolute terms, the presence of hydrocephalus did not markedly influence the polygenic risk score in autism spectrum disorder patients. This may indicate overlapping genetic architectures or other common aetiology. 
It is well known that autism spectrum disorder exhibits considerable comorbidity, most notably with epilepsy, but also with other neurodevelopmental disorders, as well as eating and sleeping disorders, gastrointestinal diseases and learning difficulties.(4) The strong association between autism spectrum disorder and hydrocephalus is in line with the findings by Lindquist et al., who reported that nine out of the 107 children born with hydrocephalus in western Sweden during the period 1989-1993 were diagnosed with autism (13\%),(13). The occurrence of autism spectrum disorder among nonmyelomeningocele hydrocephalus patients was as high as $20 \%$.

Recent developments support a significant role of abnormal CSF dynamics in the development of autism spectrum disorder. Thus Shen et al. demonstrated increased amounts of extra-axial CSF in children with autism spectrum disorder and age from 6 months to 4 years of age, regardless of familial risk.(6-9) Shen et al. concluded that "it is not clear whether increased CSF in the subarachnoid space may directly impact brain development and contribute to the pathology of psychiatric disorders such as ASD, or is an epiphenomenon that reflects another underlying aetiology."(8)

Our finding, as presented in Fig. 1, that autism spectrum disorder-polygenic risk score seem to be similar in autism spectrum disorder patients irrespective of the presence of hydrocephalus further supports this notion of a shared aetiology as the separate genetic risk factors for autism spectrum disorder tend to have an additive effect.(25) But we do not have the statistical power to say so with any certainty from this finding alone. Therefore, the indication of a shared aetiology between autism spectrum disorder and hydrocephalus relies on different arguments: First, the very strong association presented in this study, 2) the above-mentioned studies by Shen et al. on CSF abnormalities in patients with autism spectrum disorder, and 3) previously reported findings that non-syndromic hydrocephalus is associated with genetic variants affecting the Wnt signaling pathway, and that Megalencephaly-polymicrogyriapolydactyly-hydrocephalus syndromes 1-3 affect components of the PI3K-AKT-mTOR pathway.(26) Both of these signalling pathways have later shown to be implicated in autism spectrum disorder as well.(27) Together, these arguments point towards shared genetic architectures of autism spectrum disorder and hydrocephalus, at least in a subset of patients.

An important consideration is that the association observed in this study between autism spectrum disorder and hydrocephalus could also rely on a causal association, meaning that patients with hydrocephalus may develop autism spectrum disorder symptoms due to the brain damage caused by pressure to the periventricular structures in the brain. A strong argument against such a causal relation is that far from all patients with congenital hydrocephalus display symptoms of autism spectrum disorder, also according to the above-mentioned study by Lindquist et al.(13).

Another concern is that the association may be explained by surveillance bias, a potential limitation of this study design, e.g. patients with severe psychiatric disorders may have a higher probability of undergoing brain imaging studies than background population controls and therefore incidental findings of hydrocephalus could be more likely in these patients. However, we did not find evidence of any accoriation hotween hudronenhaluc and nther severe psychiatric disorders such as schizophrenia, major Loading [MathJax]/jax/output/CommonHTML/jax.js

Page $10 / 17$ 
depressive disorder, or bipolar disorder, which argues against surveillance bias. We interpret the borderline significant association between ADHD and hydrocephalus $(p=0.07)$ mainly to be the result of a substantial common genetic variant correlation between ASD and ADHD, and the recently documented overlap in genes affected by protein-truncating variants in ASD and ADHD. $(14,28)$ The ASD diagnosis in the Danish Psychiatric Central Register has been validated.(29)

Finally, one could argue that patients with autism spectrum disorder are more likely to undergo brain imaging studies than patients with the other psychiatric disorders investigated in this study. But the very strong association observed in this study (OR: $3.77,95 \% \mathrm{Cl} 2.48-5.78)$ is unlikely to be explained by incidental findings of hydrocephalus, which generally is a rare disease seen in 1.08/1,000 in children up to two years of age in Denmark.(10) Thus, we believe the effect of surveillance bias has limited impact on the results. On the contrary, the presence of autism spectrum disorder may have blurred the clinical signs of mild to moderate hydrocephalus, and vice versa. If this is the case, the association would be even stronger.

Another limitation is the potential for misclassification of diagnoses in a register-based study. However, autism spectrum disorder and hydrocephalus were the main diagnoses of interest and are two relatively distinct clinical diagnoses, so we believe the misclassification is limited. We deliberately chose to include congenital hydrocephalus and those of the acquired cases that are also considered to have a genetic predisposition, such as post traumatic or post meningitis hydrocephalus cases. We consider that environmental, causative factors, such as head trauma or meningitis, may require the interaction of a genetic factor, in order to manifest acquired hydrocephalus. This would explain why hydrocephalus after head trauma and meningitis is rare, also in our cohort. Other common causes for secondary hydrocephalus are subarachnoid haemorrhage from intracranial aneurysms and intracerebral hematoma (stroke), but our cohort members have not yet reached the typical age for stroke as they are only up 35 years old. We deliberately did not include hydrocephalus related to birth trauma (P10x, P91.7) and spontaneous perinatal intracranial haemorrhage (DP52), because they have such obvious acquired aetiologies. Also, we did not include normal pressure hydrocephalus (DG91.2), which occurs only in the elderly.

This study has several strengths, the most important is the case-cohort design, which is less prone to the biases of concern in conventional case-control studies, because the cohort is randomly selected from the entire population-based birth cohort and therefore representative for the entire Danish background population. The design also minimized loss to follow-up and missing data as reporting of diagnoses for each hospital contact to The National Patient Register is mandatory. The fact that the study is based on nationwide data, not data for a specific region or hospital only, supports the external validity of the results.

\section{Conclusions}


Irrespective of the cause of the association, our findings have clinical consequences as our data suggest that in patients with either disease; the significant risk of the presence of the other should be considered. Thus, patients diagnosed with autism spectrum disorder should be evaluated with imaging studies of the brain on a routine basis. Timely neurosurgical treatment of hydrocephalus when both conditions are present will improve the environment for the per se challenged brain development. As for future research, further clarification of the genetic aetiology of both diseases and potential shared genetic landscapes may help in identifying other common genetic causes or pathways than the ones mentioned above. This may furthermore help in elucidating the role of abnormal CSF dynamics in the pathogenesis of both autism spectrum disorder and hydrocephalus, which ultimately opens for new potential targets for medical therapy.

\section{Abbreviations}

CSF

cerebrospinal fluid

MRI

Magnetic Resonance Imaging

\section{Declarations}

\section{Ethics approval}

The study was approved by the Scientific Ethics Committees of the Central Denmark Region (www.komite.rm.dk) (J.nr.: 1-10-72-287-12) and the Danish Data Protection Agency (www.datatilsynet.dk) (J.nr.: 2012-41-0110). The Danish Neonatal Screening Biobank approved the use of the DBS samples. Detailed information on governance and ethics in the iPSYCH cohort is available at the iPSYCH website (www.ipsych.au.dk).

\section{Consent for publication}

Not applicable as no personally identifiable data is available and results are presented as aggregated data.

\section{Availability of data and materials}

The data that support the findings of this study are available from iPSYCH consortium, but restrictions apply to the availability of these data, which were used under license for the current study, and so are not publicly available. Data are however available from the authors upon reasonable request and with permission of the iPSYCH consortium. 
The authors declare that they have no competing interests.

\section{Funding}

The iPSYCH study was funded by The Lundbeck Foundation Initiative for Integrative Psychiatric Research (www.iPSYCH.au.dk). This research has been conducted using the Danish National Biobank resource, supported by the Novo Nordisk Foundation. Data handling and analysis on the GenomeDK HPC facility was supported by NIMH (1U01MH109514-01 to ADB). High-performance computer capacity for handling and statistical analysis of iPSYCH data on the GenomeDK HPC facility was provided by the Center for Genomics and Personalized Medicine, Aarhus University and Central Region Denmark, and Centre for Integrative Sequencing, iSEQ, Aarhus University (grant to ADB). Furthermore, the project was supported by grants from the Fonden til Lægevidenskabens Fremme and the Dagmar Marshalls Foundation. All are purely philanthropic foundations. The funders had no role in the design of the study, data collection, analysis, interpretation of data, or in writing the manuscript.

\section{Authors' contributions}

Tina N. Munch: Study concept and design, study supervision, interpretation of results, wrote the manuscript, critical revision of the manuscript for important intellectual content, and approval of the final manuscript.

Paula Hedley: Study concept and design, interpretation of results, co-wrote the manuscript, critical revision of the manuscript for important intellectual contentand approval of the final manuscript.

Michael Christiansen: Study concept and design, study supervision, interpretation of results, co-wrote the manuscript, critical revision of the manuscript for important intellectual content, and approval of the final manuscript.

Christian Munch Hagen: Acquisition of data, statistical analysis, critical revision of the manuscript for important intellectual content, and approval of the final manuscript.

Jakob Grove: Statistical analysis, critical revision of the manuscript for important intellectual content, and approval of the final manuscript.

Anders Børglum: Acquisition of data, critical revision of the manuscript for important intellectual content, and approval of the final manuscript.

MeretheNordentoft: Acquisition of data, critical revision of the manuscript for important intellectual content, and approval of the final manuscript.

Marie Bækvad Hansen: Acquisition of data, critical revision of the manuscript for important intellectual content, and approval of the final manuscript. 
Jonas Bybjerg-Grauholm:Acquisition of data, critical revision of the manuscript for important intellectual content, and approval of the final manuscript.

Preben B. Mortensen: Acquisition of data, critical revision of the manuscript for important intellectual content, and approval of the final manuscript.

Thomas M. Werge: Acquisition of data, critical revision of the manuscript for important intellectual content, and approval of the final manuscript.

Mads Melbye: Interpretation of results, critical revision of the manuscript for important intellectual content, and approval of the final manuscript.

David M. Hougaard: Acquisition of data, critical revision of the manuscript for important intellectual content, and approval of the final manuscript.

\section{Acknowledgements}

Not applicable.

\section{References}

1. Parner ET, Thorsen P, Dixon G, de Klerk N, Leonard H, Nassar N, et al. A comparison of autism prevalence trends in Denmark and Western Australia. J Autism Dev Disord. 2011;41(12):1601-8.

2. Schendel DE, Thorsteinsson E. Cumulative Incidence of Autism Into Adulthood for Birth Cohorts in Denmark, 1980-2012. JAMA. 2018;320(17):1811-3.

3. Robinson S, Howlin P, Russell A. Personality traits, autobiographical memory and knowledge of self and others: A comparative study in young people with autism spectrum disorder. Autism. 2017;21(3):357-67.

4. Lai MC, Lombardo MV, Baron-Cohen S. Autism Lancet. 2014;383(9920):896-910.

5. Leyfer OT, Folstein SE, Bacalman S, Davis NO, Dinh E, Morgan J, et al. Comorbid psychiatric disorders in children with autism: interview development and rates of disorders. J Autism Dev Disord. 2006;36(7):849-61.

6. Shen MD. Cerebrospinal fluid and the early brain development of autism. J Neurodev Disord. 2018;10(1):39.

7. Shen MD, Kim SH, McKinstry RC, Gu H, Hazlett HC, Nordahl CW, et al. Increased Extra-axial Cerebrospinal Fluid in High-Risk Infants Who Later Develop Autism. Biol Psychiatry. 2017;82(3):18693.

8. Shen MD, Nordahl CW, Li DD, Lee A, Angkustsiri K, Emerson RW, et al. Extra-axial cerebrospinal fluid in high-risk and normal-risk children with autism aged 2-4 years: a case-control study. Lancet 
9. Shen MD, Nordahl CW, Young GS, Wootton-Gorges SL, Lee A, Liston SE, et al. Early brain enlargement and elevated extra-axial fluid in infants who develop autism spectrum disorder. Brain. 2013;136(Pt 9):2825-35.

10. Schmidt LB, Corn G, Wohlfahrt J, Melbye M, Munch TN. School performance in children with infantile hydrocephalus: a nationwide cohort study. Clin Epidemiol. 2018;10:1721-31.

11. Marino MA, Morabito R, Vinci S, Germano A, Briguglio M, Alafaci C, et al. Benign external hydrocephalus in infants. A single centre experience and literature review. Neuroradiol J. 2014;27(2):245-50.

12. Tucker J, Choudhary AK, Piatt J. Macrocephaly in infancy: benign enlargement of the subarachnoid spaces and subdural collections. J Neurosurg Pediatr. 2016;18(1):16-20.

13. Lindquist B, Carlsson G, Persson EK, Uvebrant P. Behavioural problems and autism in children with hydrocephalus: a population-based study. Eur Child Adolesc Psychiatry. 2006;15(4):214-9.

14. Grove J, Ripke S, Als TD, Mattheisen M, Walters RK, Won H, et al. Identification of common genetic risk variants for autism spectrum disorder. Nat Genet. 2019;51(3):431-44.

15. Sullivan PF, Geschwind DH. Defining the Genetic, Genomic, Cellular, and Diagnostic Architectures of Psychiatric Disorders. Cell. 2019;177(1):162-83.

16. Kousi M, Katsanis N. The Genetic Basis of Hydrocephalus. Annu Rev Neurosci. 2016;39:409-35.

17. Furey CG, Zeng X, Dong W, Jin SC, Choi J, Timberlake AT, et al. Human Genetics and Molecular Mechanisms of Congenital Hydrocephalus. World Neurosurg. 2018;119:441-3.

18. Andersen TF, Madsen M, Jorgensen J, Mellemkjoer L, Olsen JH. The Danish National Hospital Register. A valuable source of data for modern health sciences. Dan Med Bull. 1999;46(3):263-8.

19. Norgaard-Pedersen B, Hougaard DM. Storage policies and use of the Danish Newborn Screening Biobank. J Inherit Metab Dis. 2007;30(4):530-6.

20. Mors O, Perto GP, Mortensen PB. The Danish Psychiatric Central Research Register. Scand J Public Health. 2011;39(7 Suppl):54-7.

21. Mainz J, Hess MH, Johnsen SP. The Danish unique personal identifier and the Danish Civil Registration System as a tool for research and quality improvement. Int J Qual Health Care. 2019;31(9):717-20.

22. Identification of risk. loci with shared effects on five major psychiatric disorders: a genome-wide analysis. Lancet. 2013;381(9875):1371-9.

23. Pedersen CB, Bybjerg-Grauholm J, Pedersen MG, Grove J, Agerbo E, Baekvad-Hansen M, et al. The iPSYCH2012 case-cohort sample: new directions for unravelling genetic and environmental architectures of severe mental disorders. Mol Psychiatry. 2018;23(1):6-14.

24. Borgan O, Langholz B, Samuelsen SO, Goldstein L, Pogoda J. Exposure stratified case-cohort designs. Lifetime Data Anal. 2000;6(1):39-58.

25. Weiner DJ, Wigdor EM, Ripke S, Walters RK, Kosmicki JA, Grove J, et al. Polygenic transmission dicenuilihrium nnfirmc that nnmmnn and rare variation act additively to create risk for autism Loading [MathJax]/jax/output/CommonHTML/jax.js 
spectrum disorders. Nat Genet. 2017;49(7):978-85.

26. Ekici AB, Hilfinger D, Jatzwauk M, Thiel CT, Wenzel D, Lorenz I, et al. Disturbed Wnt Signalling due to a Mutation in CCDC88C Causes an Autosomal Recessive Non-Syndromic Hydrocephalus with Medial Diverticulum. Mol Syndromol. 2010;1(3):99-112.

27. Oron O, Elliott E. Delineating the Common Biological Pathways Perturbed by ASD's Genetic Etiology: Lessons from Network-Based Studies. Int J Mol Sci. 2017;18(4).

28. Satterstrom FK, Walters RK, Singh T, Wigdor EM, Lescai F, Demontis D, et al. Autism spectrum disorder and attention deficit hyperactivity disorder have a similar burden of rare protein-truncating variants. Nat Neurosci. 2019;22(12):1961-5.

29. Lauritsen MB, Jorgensen M, Madsen KM, Lemcke S, Toft S, Grove J, et al. Validity of childhood autism in the Danish Psychiatric Central Register: findings from a cohort sample born 1990-1999. J Autism Dev Disord. 2010;40(2):139-48.

\section{Figures}

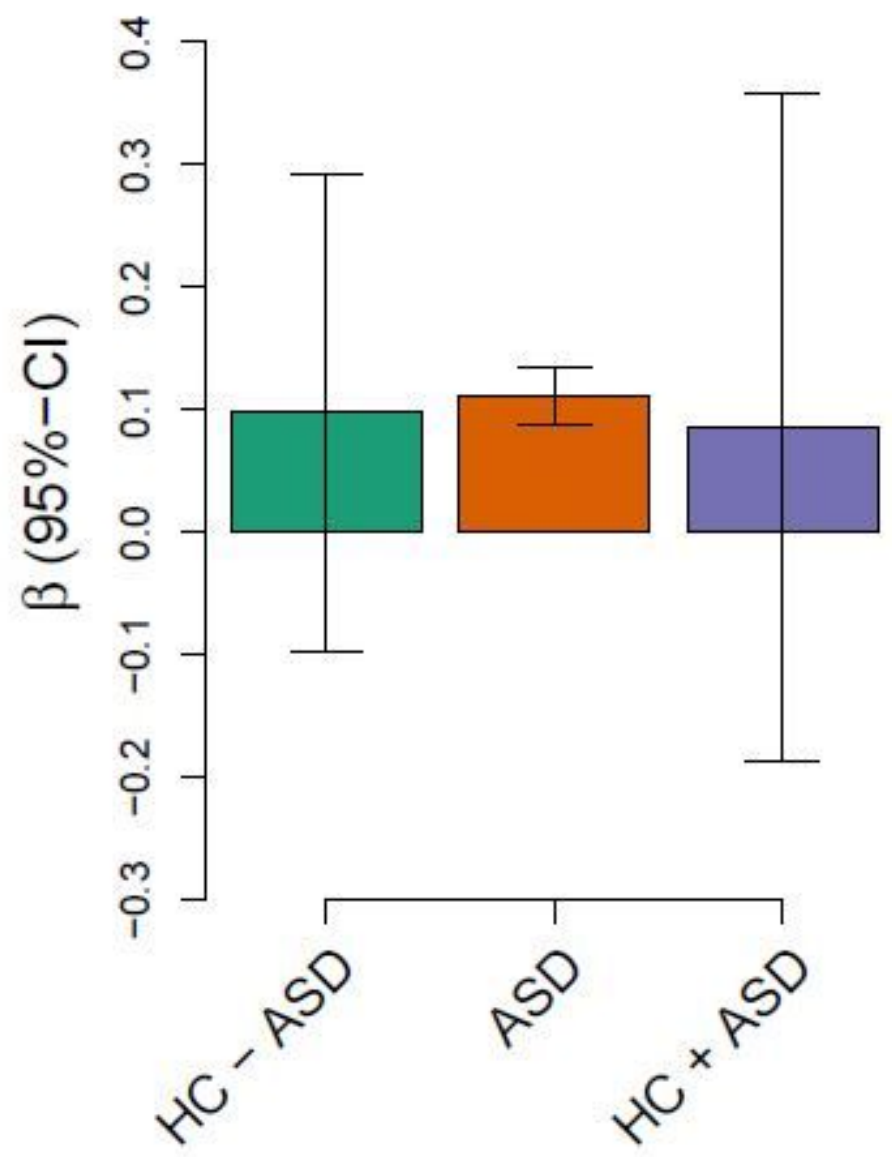

Figure 1 
Profiling autism spectrum disorder-polygenic risk scoreload in Autism Spectrum Disorder (ASD) Hydrocephalus (HC) $(n=11,716), H C+\operatorname{ASD}(n=52)$, and HC - ASD $(n=102)$ cases, using individuals without HC or ASD as reference $(n=21,848)$. Bars show coefficients from linear regression of normalizedautism spectrum disorder-polygenic risk scorescores adjusted for analytical batches and principal components. No significant group effect was found.

\section{Supplementary Files}

This is a list of supplementary files associated with this preprint. Click to download.

- Supplementarymaterial.docx 